\title{
LETTRÉS ET RÉSEAUX SAVANTS À LA COUR D’HÉRODE
}

\author{
Édith PARMENTIER \\ Maître de conférences habilitée à diriger \\ des recherches en histoire grecque \\ Université d'Angers \\ UMR 8167 Orient \& Méditerranée / \\ FRE CERHIO-TEMOS \\ edith.parmentier@univ-angers.fr
}

RÉSUMÉ

Les savants qu'Hérode fit venir à Jérusalem projettent I'image d'un roi lettré, dont la cour était le point de convergence de trois cultures : judaïque, hellénique et romaine. Ce pluralisme est d'abord attesté par les édifices culturels, théâtres et bibliothèques, dont Hérode dota son royaume ; il est ensuite confirmé par la prosopographie du cercle royal, qui dénote le transfert d'une partie du judaïsme alexandrin à Jérusalem. La cour de Judée était même assez brillante pour attirer des intellectuels venus de tout le monde gréco-romain, car le roi

MOTS-CLÉS

Bibliothèques, diplomatie,

Hérode,

Jérusalem,

judaïsme alexandrin,

pluralisme culturel,

prosopographie,

théâtres,

transferts culturels. avait fait le choix de l'ouverture méditerranéenne. Cette mixité culturelle ressort notamment de la création d'un corps diplomatique au service de la monarchie hérodienne, formé d'intellectuels gréco-romains.
Scholars brought to Jerusalem by Herod project the picture of a literate king, whose court was the focal point of Jewish, Greek and Roman cultures. This pluralism is first attested by the cultural buildings, theatres and libraries, Herod built in his kingdom. Then, a prosopographical investigation of the royal circle indicates a transfer of part of Alexandrian Judaism to Jerusalem. The court of Judea was bright enough to attract intellectuals from the whole Greco-Roman world, as the king had made the choice of Mediterranean openness. The creation of a diplomatic corps, made up of Greco-Roman intellectuals in the service of the Herodian monarchy, brings out this cultural mix.

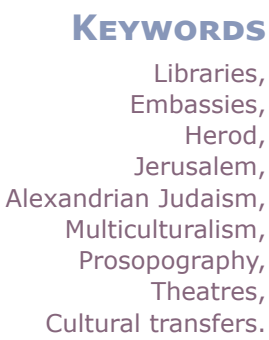


Hérode a voulu passer à la postérité comme un roi lettré : il écrivit ses propres Mémoires [1], tout en faisant rédiger I'histoire de son règne [2]. D'origine nabatéenne par sa mère, il avait été élevé dans le

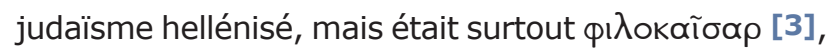
car il devait tout à l'Empire romain, qui I'avait nommé roi en lui accordant le privilège exceptionnel de désigner lui-même ses successeurs [4]. Le philhellénisme d'Hérode a été mis en évidence par la grande monographie qui lui a été consacrée dans les années soixante et qui comportait un chapitre intitulé À I'ombre de la culture et de la société gréco-romaines [5]. Plus récemment, une étude de l'image publique d'Hérode, telle que la projettent ses édifices et ses monnaies [6], a orienté I'historiographie hérodienne vers le multiculturalisme. L'approche sociologique complétant celle de l'anthropologie, le philhellénisme a fait l'objet d'études prosopographiques sur la composition de l'entourage royal [7]. La cour d'Hérode apparaît désormais comme le point de convergence de trois cultures : judaïque, hellénique et romaine. L'image du roi lettré projetée par le cercle des savants qu'Hérode fit venir dans ses palais pour éduquer ses fils - et dont il s'entoura pour se cultiver lui-même, se prenant de passion pour I'histoire après avoir abandonné successivement la philosophie et la rhétorique [8] - fait ressortir cette mixité, qu'attestent aussi les édifices culturels, théâtres et bibliothèques, dont il dota son royaume.

[1] ]ACOBY, FGrHist 236 (Flavius Josèphe, AJ, 15, 164177). Voir Geiger 2009, RolLer 2004, p. 177-185.

[2] Les Histoires de Nicolas de Damas faisaient une chronique du règne d'Hérode qui a survécu à travers les récits de Flavius Josèphe dans la Guerre des Juifs (ciaprès $G J$ ) et les Antiquités juives ( $A J)$. Une Histoire du roi Hérode, presque entièrement disparue, fut aussi rédigée par Ptolémée d'Ascalon (FGrHist 199 ; GLAJJ, n LII).

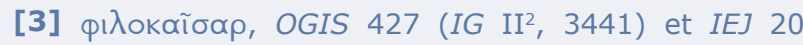
(1970), p. 97-98.

[4] GJ, I, $454 ; A J, 15,343 ; A J, 16,129$. Voir SCHALIT 2001 , p. 595. Flavius Josèphe assure qu'« Hérode tenait le deuxième rang dans l'affection d'Auguste, immédiatement après Agrippa »(GJ, I, $400 ; A J, 15,361)$. Il faut cependant nuancer cette affirmation, car le dédain l'emporte sur l'estime dans le jugement d'Auguste, si I'on se rappelle la cruelle plaisanterie qu'il aurait faite sur Hérode, selon Macrobe (Saturnales, II, 4, 11) : «Quand Auguste apprit que parmi les enfants âgés de moins de deux ans qu'Hérode, roi de Judée, avait fait tuer en Syrie, son propre fils aussi avait été exécuté, il déclara qu'il valait mieux être le porc d'Hérode que son fils »([Augustus] cum audisset inter pueros quos

\section{HÉRODE AU THÉÂTRE}

C'est dans la sphère culturelle de I'hellénisme que l'actualité archéologique conduit d'abord à placer Hérode. Dans le palais-forteresse qu'il se fit construire à douze kilomètres de Jérusalem et où il installa son mausolée - I'Hérodion - les fouilles ont récemment mis au jour un petit théâtre privé creusé au flanc de la colline [9]. Dans les dépendances (fig. 1), un graffito consigne le premier adieu d'Hector à sa mère Hécube dans I'Iliade, avant la grande scène des portes Scées : « Ne m'offre pas de vin, noble mère [10] ».

Ce graffito montre que les poèmes homériques restaient d'usage courant dans les symposia au Proche-Orient, comme dans toute la tradition grecque. L'importance prise par l'érudition homérique dans le judaïsme hellénisé et dans la littérature de cour est attestée par des œuvres comme celle du poète Théodotos, auteur d'une épopée Sur les Juifs composée à l'époque hasmonéenne, dans le dernier quart du $\mathrm{II}^{\mathrm{e}}$ siècle av. J.-C. : le poète y développe des thèmes judaïques dans une expression formelle typiquement grecque, avec un langage, un style et une prosodie homériques [11]. À l'époque hérodienne, I'hellénisation peut même être le fait de juifs de la caste sacerdotale ou de stricte observance, jusque dans la bibliothèque sectaire de Qumrân, qui compte 24 ou 25 manuscrits grecs, datés entre le

in Syria Herodes rex Iudaeorum intra bimatum iussit interfici filium quoque eius occisum, ait melius est Herodis porcum esse quam filium).

[5] SCHALIT 2001, p. 403-450.

[6] JACOBSON 1988. On retient ensuite notamment GEIGER 2002, p. 236-238, GÜNTHER 2005, p. 193-233, RoccA 2008, p. 36-52.

[7] Études initiées par WACHOLDER 1961 et poursuivies par ROLLER 1998, p. 54-65 (« The Herodian Intellectual Circle »), Geiger 2014, p. 48-52 (« The Circle of Herod »), PARMENTIER 2017.

[8] Voir I'Autobiographie de Nicolas de Damas (F 135).

[9] La construction de ce petit théâtre, dont la capacité n'excédait pas 400 spectateurs, est conforme au modèle grec classique. Voir NeTZER et al. 2010.

[10] Iliade, VI, 264. L'inscription a été découverte en 2010 : ECKER 2011, ECKER 2012.

[11] Il subsiste des fragments de cette œuvre, qui sont connus par un résumé d'Alexandre Polyhistor, transmis par Eusèbe de Césarée : ColLINS 1980, HolLADAY 1989, p. 70-72 et p. 208-210, GRUEN 1998, p. 120-127. 


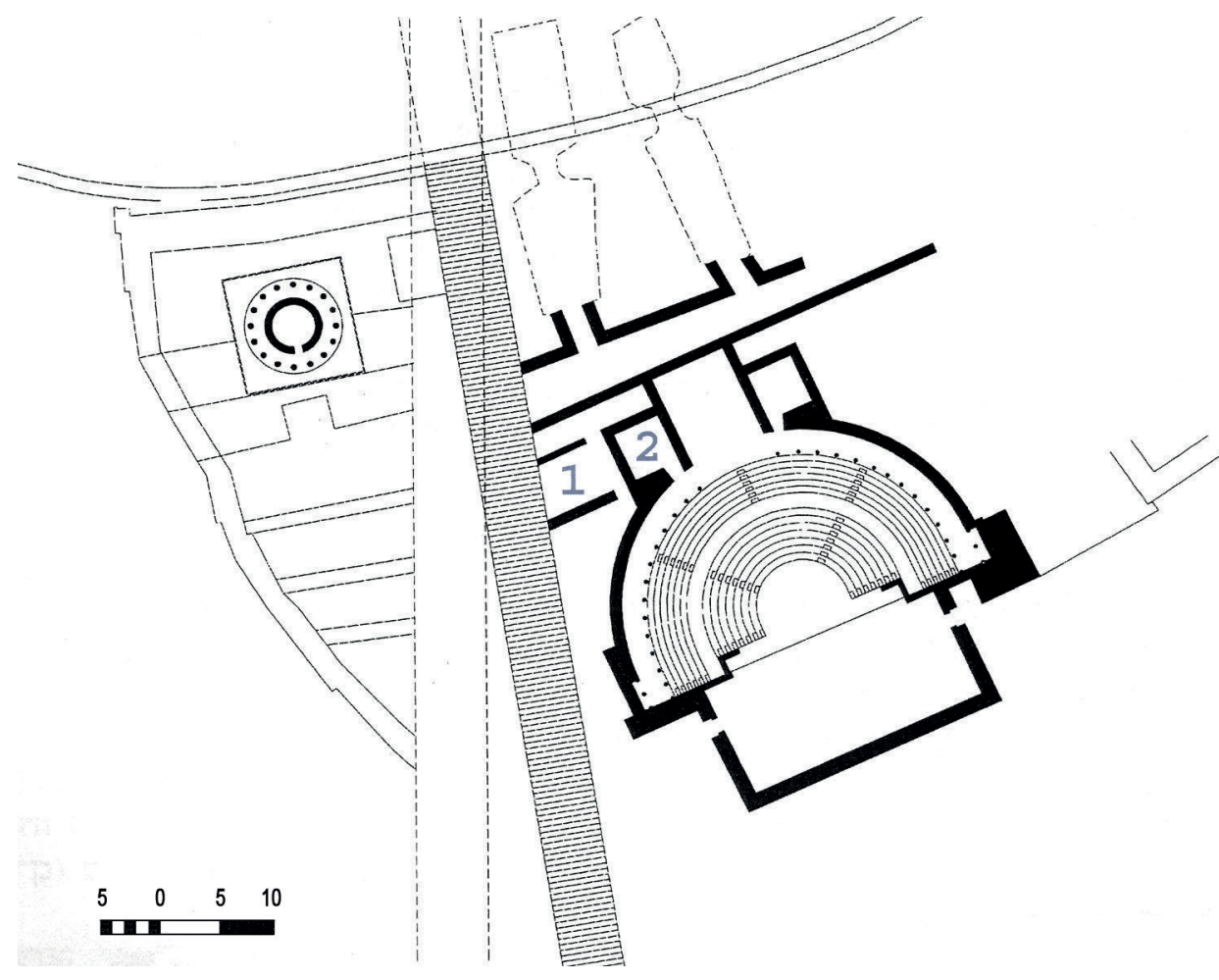

Figure 1

Plan du théâtre de I'Hérodion, avec la localisation du graffito (salle 1), d'après Ecker 2015, p. 7.
II ${ }^{\mathrm{e}}$ siècle av. J.-C. et le $\mathrm{I}^{\mathrm{er}}$ siècle ap. J.-C., sur un total de 870 manuscrits trouvés [12]. D'ailleurs, d'autres inscriptions, notamment deux alphabets grecs et deux alphabets araméens trouvés sur le site de I'Hérodion, près du théâtre (fig. 1, salle $\mathbf{2}$ ) et dans la résidence édifiée au sommet, accréditent I'hypothèse selon laquelle le bilinguisme aurait été une sorte de programme scolaire en Judée [13]. On trouve un autre témoignage épigraphique de ce bilinguisme dans les inscriptions funéraires de Jérusalem où, parmi les ossuaires d'époque hérodienne, un tiers des inscriptions sont en grec [14]. Plus tard, la popularité d'Homère reste telle que ses vers, devenus lieux communs, sont véhiculés par la littérature rabbinique [15].

Que représentait-on dans le théâtre de cour de I'Hérodion ? Le théâtre grec classique s'était largement diffusé dans les cours orientales, où il était souvent associé à des banquets. Il en était ainsi à la cour des rois parthes et des rois d'Arménie : Plutarque raconte

[12] HENGEL 1996, p. 258-294 (« Qumran und der Hellenismus »), VANDERKAM 2001.

[13] ECKER 2015. Les deux alphabets grecs et les deux alphabets araméens sont peut-être des exercices d'écoliers ; ils ont été trouvés non loin du vers d'Homère, sur un mur de la cour du palais, et étaient gravés sur un ostracon.

[14] Il s'agit d'inscriptions funéraires privées et les trois-quarts des ossuaires n'en portent pas. Mais parmi les 233 ossuaires inscrits, 73 portent des inscriptions grecques et 14 ou 15 des inscriptions bilingues (en grec et en araméen). Voir RAHMANI 1994, p. 12, VAN DER HORST 2001. qu'au moment de la défaite de Crassus à la bataille de Carrhes, en 53,

« les deux rois [Tigrane et Orodès] s'offraient I'un à l'autre fêtes et banquets, où I'on introduisait souvent I'audition d'œuvres venues de Grèce [...]. Lorsqu'on apporta la tête de Crassus à I'entrée du palais, les tables venaient d'être enlevées et un acteur tragique nommé Jason, originaire de Tralles, chantait le rôle d'Agavé dans les Bacchantes d'Euripide [16] ».

Ce sont des représentations de ce type que suggère le dispositif architectural de l'Hérodion, où la décoration de ce qu'on appelle communément la « loge royale » du petit théâtre évoque plutôt un triclinium [17]: I'auteur du graffito significatif semble avoir trompé son ennui en écrivant sur le mur, pendant qu'un banquet se déroulait dans la salle voisine (fig. 1).

[15] Lieberman 1950, p. 105. La lecture d'Homère n'était pas interdite par les prescriptions religieuses, à condition de tourner la mythologie grecque en dérision. LIEBERMAN 1950, p. 113-114, prend pour exemple le Midrash Rabbah, qui décrit l'agilité d'Asaël, neveu de David, par une référence homérique : Asaël, célèbre pour sa rapidité à la course (2Sa 2,18), « courait par-dessus les épis de maïs sans les briser » (Kohelet Rabbah, 9, 11). C'est une citation exacte de I'Iliade, XX, 227, décrivant la course des chevaux divins d'Erichtonios.

[16] Plutarque, Vie de Crassus, 33, 1-6.

[17] Netzer et al. 2013, p. 155, Vermes 2014, p. 78. 
Il subsiste un autre exemple des théâtres royaux d'Hérode [18], celui qui fut construit à l'entrée du palais de Césarée, ouvert sur la mer et pouvant accueillir 3500 à 4000 spectateurs [19]. Hérode a encouragé le théâtre juif hellénophone qui se pratiquait depuis le $\mathrm{II}^{\mathrm{e}}$ siècle à Alexandrie, où I'on a conservé une Sortie d'Égypte imitée des Perses d'Eschyle, œuvre d'un pseudo-Ézéchiel [20]. Pour la cour de Jérusalem, on dispose d'un témoignage explicite dans I'autobiographie de Nicolas de Damas, qui y arriva probablement comme précepteur des enfants royaux, en qualité de philosophe [21] : aristotélicien réputé et - dit-il - «talentueux dans les domaines de la rhétorique, de la musique, de l'étude des sciences et de I'ensemble de la philosophie [22] », Nicolas abandonna la philosophie pour I'histoire afin de répondre aux désirs d'Hérode [23]. Les goûts du roi furent sans doute aussi l'occasion pour Nicolas de revenir au théâtre, art qu'il avait pratiqué au début de sa carrière en « composant des tragédies et des

[18] L'historiographie hérodienne en compte souvent davantage, car elle rassemble tous les édifices de spectacle dans une rubrique générale de cultural buildings. Pour notre part, nous ne considérons pas les hippodromes, les stades et les amphithéâtres comme des bâtiments « culturels » (voir à ce sujet HARRIS 1976, p. 32-35). Il est possible qu'un second théâtre ait été édifié à l'Hérodion, mais s'il a existé c'était une structure provisoire de bois, réalisée à l'occasion des funérailles d'Hérode (PATRICH 2009, p. 192-193, voir aussi WeISS 2015). En revanche, ce qu'on appelle le « théâtre » de Jéricho n'en est pas un, car il y manque une scène : devant la cavea, dont les gradins de terre pouvaient accueillir 3000 spectateurs, c'est une piste de course qui s'étendait, longue de plus $300 \mathrm{~m}$ et entourée de portiques. Par ailleurs, la documentation archéologique n'a fourni aucun indice sur l'éventuel emplacement d'un théâtre hérodien à Jérusalem ; l'existence d'un tel édifice n'est même pas attestée et les restitutions qui en ont été faites ne sont que des déductions tirées du récit de Flavius Josèphe, qui mentionne des spectacles musicaux donnés lors des concours institués par Hérode en 28 en I'honneur d'Auguste. Quant à la structure théâtrale - théâtre, odéon ou bouleutérion - découverte tout récemment (en 2017) à Jérusalem, au pied du Mur occidental (sous l'Arche de Wilson), elle est postérieure au règne d'Hérode cet édifice, qui ne compte que 200 places environ, fut construit pour la colonie romaine d'Aelia Capitolina.

[19] PATRICH 2011. Contrairement au théâtre grec de I'Hérodion, celui de Césarée s'inspire du modèle romain : Hérode a imité le théâtre de Pompée, construit en 55 . Ce théâtre, que Josèphe appelle aussi amphithéâtre (GJ, I, $415 ; A J, 15,341$ ) ou stade (GJ, II, $172, A J 18,57)$, était séparé de I'hippodrome par le palais.

[20] Voir LANFRANCHI 2006.

[21] Voir SCHNEIDER 2005.

[22] Nicolas de Damas, Autobiographie, F 132 (Souda, s.v. Nikolaos).

[23] « Après s'être passionné pour la philosophie, Hérode y renonça, comme il arrive d'ordinaire à ceux à qui le pouvoir procure une foule d'avantages qui les divertissent. Il se prit d'une passion nouvelle pour la comédies réputées [24]» : il est en effet l'auteur d'une pièce intitulée Suzanne [25], dont le sujet est inspiré par un court roman hellénistique ajouté en appendice au livre de Daniel, dont nous sont parvenues deux versions grecques sensiblement différentes et qui représentent sans doute le développement d'un original sémitique [26]. L'héroïne, épouse exemplaire, apparaît comme un modèle pour les femmes juives et l'intrigue apporte la preuve que Dieu n'abandonne pas ses fidèles dans l'épreuve, en l'occurrence les poursuites injustifiées dont Suzanne a été I'objet de la part de vieillards lubriques, qui ont failli la condamner à mort. Outre Suzanne, pièce qui n'est adaptée qu'à un public juif et connaisseur de la Bible [27], on possède aussi de Nicolas de Damas l'extrait d'une comédie qui met en scène un flatteur, c'est-à-dire un courtisan [28] : le sujet de cette pièce au titre inconnu incite à penser qu'elle pourrait bien avoir eu pour commanditaire le même protecteur royal, Hérode [29].

rhétorique et réclama à Nicolas d'en faire avec lui, ce qu'ils firent. Puis une autre passion s'empara de lui, celle de I'histoire, dont Nicolas lui avait dit le plus grand bien en lui expliquant que c'était la science de la politique par excellence et qu'il y avait de l'utilité pour un roi à connaître les événements et les actions du passé. Hérode se tourna donc vers I'histoire avec ardeur en poussant Nicolas à écrire un ouvrage historique » (Nicolas de Damas, Autobiographie, F 135).

[24] Nicolas de Damas, Autobiographie, F 132.

[25] Le drame de Nicolas intitulé Suzanne n'est connu que par son titre, cité par un scholiaste byzantin qui mentionne «l'auteur du drame de Suzanne qui est, je pense, le Damascène, ainsi qu'il apparaît d'après le titre » (Eustathe de Thessalonique, Commentaire sur Denys le

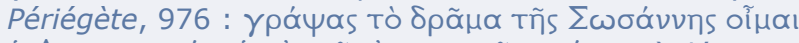

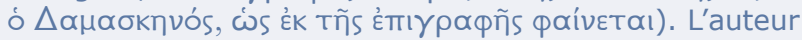
étant désigné par son ethnique dans cette formule, on attribue souvent la pièce à Jean de Damas (GGM II, p. 387, n. 18). Or, Jean de Damas est un père de I'Église du VII ${ }^{\mathrm{e}}$ siècle qui n'a pas écrit de théâtre, mais seulement des Carmina (CPG III, p. 522, n. 8070 ; PG 96, 818-856). L'attribution en faveur de Nicolas est plus vraisemblable, puisqu'il est le seul auteur damascène connu à « avoir composé des tragédies et des comédies réputées ».

[26] Daniel 13, 1-44.

[27] Ainsi Goodman 2011, p. 410, cite I'histoire de Suzanne et des vieillards comme le sujet possible d'une comédie, tout en soulignant qu'aucun témoignage explicite ne confirme l'existence de représentations théâtrales de tragédies pour les Juifs de Jérusalem. La pièce de Nicolas de Damas, si I'on admet son attribution, apporte donc un élément nouveau à ce dossier.

[28] Fragment transmis par Stobée, III 14, 7 [Sur la flatterie], 7 (PCG, vol. 7, p. 51-54, Nikolaos F 1). L'attribution des deux pièces au même auteur, mais sans I'identification avec Nicolas de Damas, est postulée par Felix Jacoby, FGrHist II C, p. 290 (commentaire de FGrHist $90 \mathrm{~F}$ 132).

[29] NeSSELRATH 1990, p. 315-317, PARMENTIER 2015, p. $23-24$, n. 20. 
La présence de ce lettré - philosophe, historien et dramaturge - à la cour de Jérusalem contribua à l'enrichissement d'autres édifices culturels, construits pour abriter les collections et les bibliothèques royales. Hérode possédait une collection d'œuvres d'art, qu'il liquida dans les années 20 av. J.-C. pour trouver des fonds au moment d'une famine qui frappa le royaume, si bien qu'on n'en connaît pas la composition [30]. La présence d'une bibliothèque hérodienne est aussi avérée au palais de Massada [31], et I'on peut en inférer l'existence d'une bibliothèque à Jérusalem.

\section{LA BIBLIOTHÈQUE D'HÉRODE}

Certes, Flavius Josèphe ne parle pas de bibliothèque royale dans son abondant catalogue des réalisations architecturales d'Hérode, mais ce silence ne prouve pas qu'un tel édifice n'ait pas existé, car l'historien ne décrit les bâtiments à destination culturelle que lorsqu'ils sont le cadre de festivités et de spectacles publics. Dans toute son œuvre, la seule bibliothèque qu'il évoque est celle d'Alexandrie, qu'il mentionne à propos de la traduction de la Bible en grec par les Septante; au demeurant, la composition de la bibliothèque intéresse beaucoup moins Josèphe que la description des cadeaux envoyés à Jérusalem par Ptolémée : I'historien ne fournit aucune autre information que le nombre des volumes réunis par Démétrios de Phalère et deux paragraphes lui suffisent pour évoquer les 400000 à 500000 ouvrages, mais il en consacre vingt-sept à détailler la liste des présents [32]. Or, l'existence d'une bibliothèque royale faisait partie de I'héritage hasmonéen et, au-delà, d'un modèle créé par la royauté achéménide. La lettre festale introduite en prologue au deuxième livre des Maccabées et datée de 164 av. J.-C. rapporte la fondation d'une bibliothèque de Néhémie, qui fut dispersée lors des combats de la période maccabéenne et restaurée par Judas Maccabée [33]. On s'interroge sur le contenu de la bibliothèque hasmonéenne, présentée par l'auteur maccabéen comme un lieu de préservation de la mémoire qui, comme le feu sacré du Temple, exprime la continuité du peuple d'Israël par-delà les ruptures brutales de son histoire. Le texte décrit une collection de documents d'archives, rassemblant, entre autres,

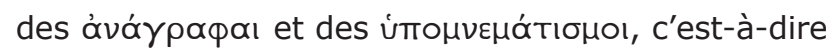
explicitement des correspondances royales ainsi que des récits historiques, mais la nature exacte du fonds hasmonéen n'est pas complètement établie, car les commentateurs de ce passage ne se sont intéressés au problème de la bibliothèque du Temple que sous I'angle de la constitution du canon biblique [34].
Quoi qu'il en soit, dès l'époque hasmonéenne l'institution et l'entretien de cette bibliothèque sont des fonctions politiques qui incombent au chef du peuple. Sous le règne d'Hérode, diverses autres bibliothèques existaient à Jérusalem [35], comme les archives publiques et les bibliothèques sectaires, notamment celle des esséniens. La nouveauté de la bibliothèque royale d'Hérode se réduit donc à sa diversité culturelle et à sa modernité. La composition peut en être partiellement reconstituée à partir des auteurs cités par Nicolas de Damas, qui les a lus et utilisés pour rédiger ses Histoires, œuvre dont Hérode était le premier destinataire [36].

Il y a d'abord un fonds classique incluant évidemment Homère et Hésiode, le théâtre d'Euripide et aussi Hérodote, Xénophon, Éphore et Polybe, ainsi qu'un certain nombre d'historiens locaux, tels Hellanicos de Lesbos, Xanthos de Lydie et Acousilaos d'Argos. Ces auteurs sont abondamment cités par Nicolas de Damas dont, par ailleurs, les philosophes de prédilection sont Aristote et Théophraste, l'auteur étant lui-même aristotélicien [37]. Même si I'on peut admettre que Nicolas connaissait les vers d'Homère et d'Euripide par cœur, la référence à des historiens locaux témoigne indirectement de la présence de leurs ouvrages dans la bibliothèque. Cependant, la place des historiens dans le catalogue ne doit pas être surévaluée, car il va de soi qu'elle est liée à la nature de l'ouvrage que Nicolas était en train de rédiger.

Un fonds orientaliste, également attesté par les emprunts de Nicolas de Damas dans ses Histoires, est bien représenté par les Persica de Ctésias, les mémoires d'Aristobule, compagnon d'Alexandre, et surtout par les Babyloniaca de Bérose, cette synthèse des traditions religieuses et historiques de la Babylonie qui était destinée aux Grecs et fut rédigée lors de l'installation du royaume séleucide [38]. S'y ajoutent le livre de Laitos qui composa des Phoinicica

[30] AJ, 15, 306.

[31] HiRSCHFELD 2006.

[32] $A J, 12,12-13$ (volumes) et 57-84 (cadeaux).

[33] 2Mac 2, 13-14. Voir NA'AMAN 2006, BASLEZ 2007, p. 228-236.

[34] Abel \& STARCKY 1961, p. 308-309, SCHWARTZ 2008, p. 166-167.

[35] RICHARDSON 1963, p. 183-191.

[36] Nicolas de Damas, Autobiographie, F 135. Voir WACHOLDER 1962, p. 81-86 (« Greek Authors in Herod's Library »).

[37] PARMENTIER \& BARONE 2011, p. XX-XXI.

[38] Sur Bérose, voir KUHRT \& SHERWIN-WHITE 1987, p. 32-56 (« Berossus' Babyloniaka and Seleucid Rule in Babylonia ») et, plus récemment, CAMPOS DAROCA 1994. 
imitant les Babyloniaca de Bérose [39], les Syriaca de Théodore de Gadara [40], ainsi que les ouvrages de Strabon, qui y était représenté à la fois par ses Histoires et par sa Géographie, tout en étant lui-même le relais d'un certain nombre d'auteurs orientaux, en particulier Timagène [41]. Cette diversité éclaire la gouvernance pluraliste qui s'était installée dans le Proche-Orient pour gérer la mosaïque de peuples et de religions coexistant dans la région. Dans ce fonds orientaliste, un certain nombre d'ouvrages concernent plus particulièrement I'histoire du judaïsme, comme les récits sur le déluge et I'histoire des Juifs par Mnaséas de Patara [42], les chroniques d'Apollodore d'Athènes sur Antiochos IV [43], I'Histoire de Poseidonios d'Apamée [44], I'Histoire des Juifs d'Alexandre Polyhistor [45] et les récits de Castor de Rhodes sur I'histoire de la Palestine [46]. On peut difficilement imaginer que les Mémoires d'Hérode lui-même n'y aient pas figuré [47], ainsi que I'Histoire d'Hérode de Ptolémée d'Ascalon, même si cet ouvrage n'apparaît pas dans les fragments conservés de Nicolas [48].

La bibliothèque d'Hérode existait donc et elle n'a cessé de s'agrandir sous son règne, comme en témoigne l'abondance d'un fonds gréco-romain contemporain. Déjà Alexandre Polyhistor était passé

[39] FGrHist $784 ;$ GLAJJ, $\mathrm{n}^{\circ}$ XXIII.

[40] FGrHist 850.

[41] FGrHist 88. Voir SIRINELLI 1993, p. 168.

[42] GLAJJ, n॰ XV (Mnaséas de Patara, vers 200 av. J.-C.).

[43] GLAJJ, n XIX (Apollodore d'Athènes).

[44] FGrHist 87 ; GLAJJ, $\mathrm{n}^{\circ} \mathrm{xXVIII.} \mathrm{Sur} \mathrm{Poseidonios}$ d'Apamée (135-51 av. J.-C.), voir SIRINELLI 1993, p. 164-165.

[45] FGrHist 273 ; GLAJJ, $\mathrm{n}^{\circ} \mathrm{xXX}$.

[46] GLAJJ, n XXXVII (Castor de Rhodes, né vers 60 av. J.-C.).

[47] FGrHist 236. Voir ROLLER 2004, p. 177-185.

[48] FGrHist $199 ;$ GLAJJ, $\mathrm{n}^{\circ}$ LII. Selon Jacoby, FGrHist II D, p. 625-626, ce Ptolémée pourrait être le frère de Nicolas de Damas ; voir aussi DiHLE 1959.

[49] Originaire d'Asie Mineure, fait prisonnier lors de la campagne de Sylla contre Mithridate VI Eupator, esclave puis affranchi à Rome, Alexandre Polyhistor (80-35 av. J.-C.) est l'auteur d'au moins 25 ouvrages. Voir SIRINELLI 1993, p. 167-168.

[50] Timagène avait été réduit en esclavage comme prisonnier en 55. Voir LAQUEUR 1936, SORDI 1982-1983.

[51] Suétone, Vie des Douze Césars, Tibère, 57.

[52] Ces fragments résultent très probablement d'une interpolation de copiste (PARMENTIER 2002).

[53] M. Vipsani Agrippae fragmenta ad Chorographiam spectantia, dans RIESE 1878. Les indications laissées par Agrippa dans cet ouvrage sont à la base de la carte du monde qu figurait sur le Portique du Champ de Mars et qui est peutêtre le modèle de la Table de Peutinger (RodDAZ 1984, p. 291-293 et p. 583-587, NICOLET 1988, p. 103-131). par Rome comme prisonnier de guerre [49]; de même, Timagène d'Alexandrie, affranchi devenu rhéteur et historien, fut protégé par Asinius Pollion et par Auguste avant de tomber en disgrâce [50] ; Théodore de Gadara, quant à lui, avait fait partie de la Maison de Tibère comme professeur de rhétorique [51]. On discute aussi de la présence, dans ce fonds, des Antiquités romaines de Denys d'Halicarnasse, dont quelques passages sont incorporés dans les Histoires de Nicolas [52]. La bibliothèque d'Hérode a également collectionné les mémoires d'hommes politiques contemporains de la fondation de l'Empire : les Commentaires de César et les Res Gestae d'Auguste, cités par Nicolas, y figuraient et I'on déduit de liens personnels et familiaux avec Hérode que la Chorographie d'Agrippa, qui visita le roi en 15 av. J.-C., était représentée dans la bibliothèque [53], ainsi que I'Histoire romaine et les mémoires ethnographiques du roi Juba de Maurétanie, qui avait épousé la fille d'Antoine et de Cléopâtre [54]. Les œuvres d'Archélaos de Cappadoce devaient, à plus forte raison, y trouver place, puisque sa fille Glaphyra avait épousé le fils d'Hérode, Alexandre, successeur désigné du roi de Judée [55]. Enfin, on ne saurait oublier qu'une bibliothèque antique est aussi un dépôt d'archives [56], ce qui explique l'abondance de

[54] FGrHist 275. La tradition romaine, transmise par un Aide-Mémoire scolaire composé en Maurétanie, a décerné à Juba le titre Rex literatissimus (L. Ampelius, Liber Memorialis, 38, 2). Le catalogue des œuvres de cet auteur polygraphe, qui fut éduqué à Rome comme otage pendant vingt ans avant d'être désigné roi en 25 av. J.-C., a été étudié par Roller 2003, p. 163-182 et p. 261263. Il en reste une centaine de fragments, portant sur des sujets multiples, depuis les compositions historiques comme les Antiquités romaines (ou Histoire romaine), à la manière de Denys d'Halicarnasse, jusqu'aux monographies ethno-géographiques sur l'Assyrie, la Libye, l'Arabie, en passant par des ouvrages sur la peinture, le théâtre, les analogies culturelles entre le monde grec et le monde romain, le langage en général ainsi que la botanique (traité Sur l'euphorbe). Les écrits de Juba ont été transmis pour la plupart en latin, mais furent composés en grec.

[55] FGrHist 123. Proche de Juba, à qui il est souvent comparé (Solin, 52, 18-23, Pline, Histoire naturelle, 37, 107-108), Archélaos de Cappadoce écrivit une histoire d'Alexandre le Grand, un manuel d'agriculture, un traité sur la religion et d'autres ouvrages, dont peut-être des écrits Sur les rivières et Sur les pierres (ROLLER 2003, p. 219220 , n. 52-53). Il régna de 36 av. J.-C. à 17 ap. J.-C. et maria sa fille Glaphyra à trois rois, dont deux Hérodiens : d'abord au fils d'Hérode, Alexandre, initialement désigné comme son successeur mais exécuté en 7 av. J.-C., puis à Juba, et enfin à un autre fils d'Hérode, Hérode Archélaos, qui fut son successeur effectif comme ethnarque à partir de 4 av. J.-C.. Voir SULLIVAN 1980, p. 1149-1161, SULLIVAN 1990, p. 182-185, JACOBSON 2001.

[56] Voir sur ce point COQUEUGNIOT 2013. 
documents officiels d'époque césarienne et augustéenne cités par Josèphe, qui recopie les écrits de Nicolas de Damas aux livres 14 à 17 des Antiquités juives. Compte tenu du fait que nous ne connaissons cette bibliothèque qu'à travers un de ses utilisateurs, qui était un historien et un politique, on peut sans doute conclure de cet inventaire que le pluralisme culturel qui marquait la cour de Jérusalem ne s'est pas limité à combiner, comme dans tout le Proche-Orient, un hellénocentrisme hérité et un penchant «philobarbare [57] », mais a aussi fait intervenir un tropisme romain résolument contemporain.

Il est possible que le fondateur de la première bibliothèque publique de Rome, Asinius Pollion, I'orateur, poète et historien, ait été l'hôte d'Hérode à Rome, accueillant les fils du roi dans sa maison entre 23 et $18-17$; il aurait alors joué un rôle de médiateur culturel vis-à-vis d'Hérode, de sa famille et de sa cour [58]. En effet, C. Asinius Pollion, ami d'Antoine, semble être devenu I'un des plus chauds partisans d'Hérode à partir de l'année 40 , lors de la désignation de ce dernier comme roi par le Sénat romain. Asinius Pollion était alors consul en exercice ; I'année suivante, devenu proconsul de Macédoine, il mena campagne en Syrie contre les Parthes [59], au moment même où Hérode les combattait parallèlement en Judée pour installer son royaume. C'est à la suite de son triomphe célébré sur les Parthes, en 39 ou en 38 av. J.-C., qu'Asinius Pollion fonda sa bibliothèque, en utilisant sans doute le butin de cette campagne [60]. Toutefois, cette identification a été remise en doute : plutôt que I'homme politique lui-même, l'hôte d'Hérode pourrait être un affranchi, Publius Vedius Pollion, favori d'Auguste et membre de I'ordre équestre, qui fit fortune dans l'exploitation de vignobles et le commerce de vins en Asie Mineure [61]. Ce Vedius Pollion était un ami d'Hérode, à qui il envoyait du vin des propriétés qu'il possédait en mer Égée : les fouilles de l'Hérodion ont livré 38 amphores estampillées au nom de P. VE. POL, qui ont été interprétées comme des cadeaux et comme un témoignage des relations entre Hérode et Publius Vedius Pollion [62]. L'hypothèse de liens entre les deux personnages est renforcée par un autre argument archéologique : Vedius Pollion est réputé pour avoir construit, dans sa villa du Pausilippe, un des plus anciens théâtres privés romains ; ce théâtre est le seul connu de l'époque - en-dehors de celui de la villa de l'île de Pianosa, où Agrippa Postumus fut exilé par Auguste - pour avoir été construit sur le même modèle que celui d'Hérode à l'Hérodion, avec une loge surmontant les gradins.

Ainsi Hérode a non seulement assumé un héritage judéen pour composer sa bibliothèque, mais il a probablement aussi utilisé un modèle romain de proximité. De plus, le contexte géopolitique offrait un vide à combler en Orient, après les dommages subis par les deux grandes bibliothèques de I'Orient hellénisé, celle d'Alexandrie et celle de Pergame. La bibliothèque d'Alexandrie ayant beaucoup souffert des guerres civiles et de l'incendie de 48 av. J.-C., Antoine avait tenté de la reconstituer en dépouillant la bibliothèque de Pergame, d'où il avait déplacé 200000 rouleaux [63]. Il est vraisemblable que l'incendie de la bibliothèque, puis la disparition de la cour de Cléopâtre, ont occasionné des transferts de biens culturels, comme celui qu'on constate au bénéfice de Juba de Maurétanie, et donné une importance nouvelle à la bibliothèque royale de Jérusalem. Et si la bibliothèque en a certainement profité, il semble également possible de constater une émigration des lettrés du Musée vers la cour d'Hérode.

\section{D’ALEXANDRIE À JÉRUSALEM : UNE CARTE DU MONDE LETTRÉ}

La chute de la royauté lagide, qui lança des intellectuels réputés sur les routes, entraîna-t-elle une migration massive d'Alexandrie vers Jérusalem ? L'hypothèse d'un renforcement de la présence grecque à la cour d'Hérode, par l'arrivée d'exilés alexandrins
[57] Cf. l'expression « storico ellenocentrico e filobarbaro », employée par SORDI 1982-1983 à propos de Timagène.

[58] AJ, 15, 342-343 : « Hérode décida d'envoyer ses fils Alexandre et Aristobule à Rome, pour qu'ils fréquentent Auguste. À leur arrivée, ils furent hébergés dans la maison de Pollion, un des hommes qui cultivaient l'amitié d'Hérode avec le plus d'empressement, et ils furent également admis à résider chez Auguste ». Voir Отто 1913 col. 70. L'identification de ce Pollion au nom imprécis avec Asinius Pollion est due à FELDMAN 1953.

[59] GLAJJ, $\mathrm{n}^{\circ} \mathrm{XXXVI}$, avec la bibliographie, ANDRÉ 1949, ZECCHINI 1982-1983.
[60] COARELLI 1993.

[61] Ce Pollion a été rendu célèbre par SYME 1961. Voir la notice prosopographique de KIRBIHLER 2012. L'identification de I'hôte d'Hérode avec Asinius Pollion a été a contestée de façon convaincante par BRAUND 1983 (contra, FELDMAN 1985) ; ensuite, KoKKINOS 2010, p. 214, n. 27 , a laissé la question ouverte, mais depuis lors l'archéologie semble avoir confirmé la candidature de Vedius Pollion.

[62] FINKIELSZTEJN 2006, p. 137-139, BAR-NATAN \& GÄRTNER 2015, d'après I'identification de Finkielsztejn.

[63] Plutarque, Vie d'Antoine, 58. 
après la mort de Cléopâtre, est défendue par Duane W. Roller. Cette conjecture se fonde d'abord sur le constat du caractère massif et constant de l'émigration des aventuriers et des spécialistes de haut niveau [64] : ainsi la prise du pouvoir par Ptolémée VIII en 145 av. n. è. avait déjà provoqué une émigration des intellectuels du Musée [65], dont la principale bénéficiaire fut alors Rhodes [66]. Ensuite, il faut postuler une étape intermédiaire : I'intégration d'Hérode au cercle d'Antoine lors du premier séjour qu'il fit à Rome en 40 , avant de régner ; les liens créés dans ce cadre auraient ensuite été réactivés après la disparition de la cour d'Alexandrie [67]. De fait, la prosopographie semble confirmer le transfert d'une partie du judaïsme alexandrin à la cour d'Hérode.

Un « Ami royal » du nom d'Olympos, ambassadeur d'Hérode [68], peut être identifié assez probablement à un médecin de Cléopâtre connu par Plutarque [69] : la vraisemblance est forte, car le nom d'Olympos est peu fréquent. Un autre personnage, nommé Dosithéos, qui, d'après les Mémoires d'Hérode, joua un rôle décisif à ses côtés en 30 [70], est peut-être identifiable avec l'Alexandrin Dosithéos, fils de Cleopatridès d'Alexandrie, qui négocia auprès du magistrat romain I'exemption du service militaire pour les juifs d'Ephèse, dans les années 49 [71]. Cependant, ce nom théophore est très répandu, en particulier dans le judaïsme alexandrin (Dosithéos traduit I'hébreu Nathan - «don de Dieu ») [72], et la fréquence des homonymies fragilise l'identification [73].

C'est encore une homonymie, combinée avec des rencontres événementielles possibles, qui incite à rapprocher un certain Antiphilos [74], proche d'Antipater fils aîné d'Hérode, d'un médecin d'Alexandrie qui avait eu des contacts à Rome avec la famille impériale : Antiphilos, frère d'un médecin alexandrin et probablement médecin lui-même, fit un séjour en Égypte et en rapporta un poison destiné à tuer Hérode [75].

Il est très tentant de postuler aussi l'origine alexandrine de l'un des précepteurs princiers, Andromachos [76] : conseiller et ambassadeur d'Hérode en même temps que précepteur de ses fils, Andromachos éduqua son propre fils Démétrios comme oúvtpoфos d'Alexandre, I'un des fils d'Hérode et de Mariamme, successeur désigné jusqu'à son exécution en 7 av. J.-C. [77]. La référence polythéiste du nom «Démétrios » qu'Andromachos donna à son fils exclut la possibilité qu'il ait été juif ; bien plus, le fait qu'Andromachos ait reçu le titre d'Ami royal signifie que la présence d'une élite grecque à la cour d'Hérode allait en se renforçant au cours du règne. Un autre précepteur d'Alexandre, Gemellus, offre un peu le même profil qu'Andromachos : il accompagna son élève à Rome, où les fils d'Hérode étaient éduqués, comme on l'a vu à propos de Pollion, ce qui peut appuyer I'hypothèse de sa participation au cercle d'Antoine et de relations nouées avec Hérode dans ce cadre, lors des séjours du roi à Rome ou à Alexandrie [78]. Cependant, aucun de ces éléments ne suffit pour supposer automatiquement aux précepteurs une origine alexandrine. En définitive, c'est le passage, vraisemblable sinon assuré, de Nicolas de Damas et de son frère Ptolémaios par la cour de Cléopâtre et d'Antoine, avant leur intégration
[64] Voir BASLEZ 2008, p. 211-233 (« L'émergence d'une classe politique internationale »).

[65] Selon Athénée, IV, 184 c, citant I'historien Ménéclès de Barca, « il y eut un renouveau de toute la culture au temps du Ptolémée VIII, le roi d'Égypte, surnommé à juste titre 'le Malfaisant' par les Alexandrins. En effet, il fit périr beaucoup d'Alexandrins, et en très grand nombre il exila ceux qui avaient grandi avec son frère [Ptolémée VI] : il remplit ainsi les îles et les cités de grammairiens, de philosophes, de géomètres, de musiciens, de peintres, de maîtres de gymnastique, de médecins et bien d'autres praticiens des arts. Réduits par la pauvreté à enseigner ce qu'ils savaient, ils assurèrent la formation de nombreux hommes distingués » (trad. Ch. Jacob).

[66] Voir BRINGMANN 2002.

[67] ROLLER 1998, p. 55.

[68] Olympos mène une ambassade à Rome pour annoncer à Auguste l'arrestation des princes héritiers Alexandre et Aristobule (GJ I, 538 ; AJ 16, 332). Voir SCHALIT 1968, p. 92, « Olympos».

[69] Plutarque, Vie d'Antoine, 82.

[70] GJ, I, 431-434; AJ, 15, 161-182 (Mémoires d'Hérode). SCHALIT 1968, p. 39, « Dosithéos 3 ».
[71] AJ, 14, 236-237. SCHALIT 1968, p. 39, « Dosithéos 2 ». [72] Dosithéos est un nom aussi fréquent que celui d'Alexandre : ILAN 2000, p. 273-276, ne recense pas moins de 31 Dosithéos.

[73] Envisagée par FELDMAN 1965, ad loc., I'identification entre «Dosithéos 2 » et « Dosithéos 3 » est admise par RoLLER 1998, p. 58, n. 7, mais elle n'est pas acceptée par PUCCI BEN ZEEV 1998, p. 182-185, qui considère le Dosithéos fils de Cleopatridès d'Alexandrie comme « inconnu par ailleurs »; GEIGER 2014 rejette aussi cette identification.

[74] GJ, I, 592, 598 ; AJ, 17, 70, 73, 77. Schalit 1968, p. 13, « Antiphilos ».

[75] G], I, $598 ; A J, 17,73$ et 134-135, 144. L'identification doit s'arrêter là et rien ne permet d'en déduire qu'Antiphilos descendait du peintre alexandrin du même nom, comme le suppose RoLLER 1998, p. 58, n. 4.

[76] $A J, 16,242,245$. Schalit 1968, p. 11, « Andromachos ».

[77] $A J, 16,243$.

[78] AJ, 16, 242. Schalit 1968, p. 34, « Gemellos». Cette brève mention est souvent extrapolée jusqu'à faire de Gemellus « the latin tutor » des enfants royaux. 
à la cour hérodienne, qui est à l'origine de toutes ces hypothèses [79].

En effet, Nicolas, historien et philosophe, aurait été le précepteur des enfants de Cléopâtre et d'Antoine, selon un témoignage chrétien qui, bien que tardif, semble digne de foi dans la mesure où il représente une tradition damascène locale :

« Il y avait à Damas un certain Dionysios, qui faisait partie des magistrats en vue et était issu d'une famille qui n'avait jamais cessé d'être illustre. Elle avait pour origine et pour ancêtre le philosophe Nicolaos, maître d'Hérode et précepteur des enfants d'Antoine et de Cléopâtre; à sa suite fleurirent aussi douze générations de Nicolaos, brillants philosophes qui illustrèrent la famille et la conduisirent à un degré élevé de notoriété et de célébrité [80] 》.

La question reste ouverte de savoir si c'est à Alexandrie ou à Rome qu'eut lieu ce que Joseph Geiger appelle « the second stage » de la carrière de Nicolas, devenu précepteur royal après une carrière locale de philosophe [81]. Il pourrait avoir rencontré Cléopâtre en 36 av. J.-C., lorsque celle-ci passa par Damas pour rejoindre Antoine engagé dans sa campagne contre les Parthes ; à cette date, quand Antoine donna la Cœlé-Syrie à Cléopâtre [82], elle devint reine de Damas et la cité frappa monnaie à son type. Dans ce cas, Nicolas serait demeuré à Alexandrie jusqu'à la mort de Cléopâtre et aurait partagé ses fonctions

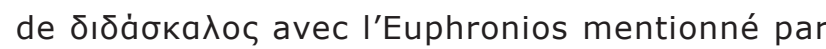
Plutarque [83]. Mais cette hypothèse est affaiblie par le fait que ni Plutarque - qui pourtant connaît Nicolas et le décrit par ailleurs comme un philosophe péripatéticien ami d'Hérode [84] -, ni aucune autre source ne citent Nicolas parmi les tuteurs et pédagogues des enfants royaux [85]. Néanmoins, il se peut que les jumeaux Alexandre Hélios et Cléopâtre Séléné, nés en 40, aient eu plusieurs précepteurs en même temps à Alexandrie ou bien qu'Euphronios y ait succédé à Nicolas. L'autre possibilité serait que Nicolas ait rempli son rôle auprès des enfants d'Antoine et de Cléopâtre plus tard, quand, après la bataille d'Actium et la mort de leur mère, ils furent emmenés à Rome pour être élevés dans l'entourage d'Octavie [86], qui aurait alors fait appel à lui comme précepteur. Cette hypothèse permettrait de rendre compte du silence des sources, notamment Plutarque, sur la présence de Nicolas à Alexandrie et, inversement, de sa présence attestée dans l'entourage d'Auguste quelques années plus tard, où les témoignages montrent que l'empereur appréciait en lui non seulement le lettré, mais le compagnon et I'ami [87]. Plutôt que d'envisager la relation entre Hérode et Nicolas comme étant issue du cercle d'Antoine, il est donc possible qu'elle se soit créée plus tard, dans I'entourage d'Auguste, peut-être quand Nicolas accompagna ce dernier en Syrie en 20 av. J.-C., au cours d'un voyage où l'empereur rencontra Hérode à Antioche [88]. Étant donné I'absence de documentation sur la carrière hérodienne de Nicolas avant l'année 14 , on peut aussi bien supposer que c'est Auguste qui le fit connaître à Hérode ou, inversement, que c'est Hérode qui le présenta à Auguste [89].

$C^{\prime}$ est encore le mouvement présumé d'une cour à I'autre, d'Alexandrie à Jérusalem, qui soutient I'hypothèse formée sur le philosophe Philostratos : sophiste de la cour de Cléopâtre, lié auparavant à Caton le Jeune, Philostratos fut disgracié et exilé en 30 av. J.-C., à la suite de la mort de la reine et de l'établissement de la domination romaine [90] ; c'est alors qu'il aurait rejoint la cour d'Hérode [91]. L'argument s'appuie sur une épigramme évoquant la relégation de Philostratos,
[79] La carrière de Ptolémaios semble suivre celle de son frère (Nicolas de Damas, Autobiographie, F 131), bien qu'il ne soit cité par Josèphe qu'après la mort d'Hérode : Ptolémaios apparaît alors lié à Antipas dans le conflit entre les successeurs du roi, tandis que Nicolas soutient Archélaos (GJ, II, $21 ; A J, 17,225$ ). SCHALIT 1968, p. 99, «Ptolémaios 8 ». Sur ce personnage, voir GAGGERO 2011.

[80] Nicolas de Damas, T 2 = Sophronios de Damas, Miracles des saints Cyr et Jean, 54. Sophronios vécut au $\mathrm{VII}^{\mathrm{e}}$ siècle et fut patriarche de Jérusalem entre 634 et 638 .

[81] Selon GEIGER 2014, p. 48, la carrière de Nicolas de Damas se serait déroulée en quatre phases : la première, locale à Damas, la deuxième à Alexandrie comme précepteur, la troisième à Jérusalem, dès les années 30 , et la quatrième à Rome après la mort d'Hérode.

[82] $A J, 15,96-105$.

[83] Plutarque, Vie d'Antoine, 72, 2.
[84] Plutarque, Propos de table, VIII, IV, 1 (Nicolas de Damas, T 10 b) et Vies parallèles, Brutus, 53, 5-7 (Nicolas de Damas, F 99).

[85] L'hypothèse de RolLeR 2010, p. 128, pour qui Nicolas aurait été le bibliothécaire de Cléopâtre, est une pure conjecture.

[86] Plutarque, Vie d'Antoine, 87, 1. Sur l'éducation de ces enfants princiers, voir ROLLER 2003, p. 76-90.

[87] Athénée (Nicolas de Damas, T 10 a) et la Souda (Nicolas de Damas, T 1) présentent Nicolas comme I'un des familiers d'Auguste.

[88] AJ, 15, 354-358.

[89] PARMENTIER \& BARONE 2011, p. XIII-XIV.

[90] Plutarque, Vie d'Antoine, 80, 3 et Caton le Jeune, 57, 4, Philostrate, Vie des Sophistes, 1, 5.

[91] Proposition faite avec précaution par GLAJJ, p. 217219, reprise avec plus d'assurance par ROLLER 1998, p. 62-63, et maintenue par GEIGER 2014, p. 49. 
due à Crinagoras de Lesbos. Cette épigramme situe le tombeau du philosophe à Ostrakina, petit village du désert maritime, proche de Gaza, étape sur la route d'Alexandrie à Jérusalem [92]. La date de la mort de Philostratos étant inconnue, on ne peut déterminer s'il mourut en chemin immédiatement après sa disgrâce ou s'il eut le temps de se réfugier à la cour d'Hérode et d'y développer une activité :

\section{«Pauvre victime de ton opulence, Philostratos, Où sont pour toi ces sceptres illustres et tes multiples succès auprès des rois \\ au milieu desquels tu as toujours tenu ta vie suspendue? \\ C'est sur les bords du Nil que tu gis, bien visible aux confins de la Judée. \\ Des étrangers se sont partagé tes œuvres, tandis que ton cadavre demeurera toujours dans l'aride Ostrakina [93] ».}

Le héros de Crinagoras étant un intellectuel d'Alexandrie, dont les œuvres furent réparties, selon I'épigramme, entre plusieurs cours étrangères, il peut fournir un exemple de transfert socio-culturel, même si la présence de livres dans différentes bibliothèques ne présuppose pas un déplacement physique de l'auteur. Toutefois, I'identification ne peut être certaine à cause de la fréquence du nom de Philostratos [94].

De la même façon, le nom grec du secrétaire d'Hérode $n^{\prime} e s t$ pas un indice suffisant pour en déduire son origine géographique et ethnique : ce $\gamma p \alpha \mu \mu \alpha \tau \varepsilon u ́ s$ nommé Diophantos, qui abusa de sa position et fut condamné pour avoir falsifié des lettres [95], porte un nom dont le composé $\Delta$ ıo- renvoie initialement à Zeus, mais la référence polythéiste a complètement disparu à l'époque ; les noms grecs théophores où l'élément divin évoque Zeus ont subi la même assimilation que les anthroponymes composés sur $\Theta \varepsilon o-$ : les noms de Diophantos, Diodotos et Zénodoros sont couramment attestés dans les milieux juifs [96].

[92] GJ, IV, 661.

[93] Anthologie Palatine, VII. Épigrammes funéraires, 645.

[94] Signalons un amalgame supplémentaire, sans fondement, avec un historien du même nom, qui écrivit sur la Phénicie et sur l'Inde - ou, avec une légère correction, sur la Judée.

[95] G], I, $529 ; A J, 16,319$.

[96] MUSSIES 1994, p. 245-246.

[97] GLAJJ, no LXXVIII, p. 218-219.

[98] GREFFCKEN 1922.
Bien que souvent fragiles, ces identifications sont assez nombreuses pour montrer qu'Hérode a puisé dans le vivier alexandrin, plus précisément dans le milieu judéo-alexandrin. De fait, la cour de Jérusalem semble avoir été assez brillante pour attirer des intellectuels venus de tout le monde gréco-romain, car la venue en Judée de visiteurs tels que le poète Crinagoras de Lesbos, I'orateur Eirénaios et I'historien et géographe Strabon lui-même est possible.

Crinagoras de Lesbos est l'auteur de l'épigramme citée plus haut sur le philosophe Philostratos, I'un des lettrés alexandrins peut-être réfugiés auprès d'Hérode [97]. Il est connu autrement comme un poète de cour augustéen [98], qui composa ses épigrammes les plus célèbres pour Cléopâtre Séléné, en I'honneur de son mariage avec Juba et de sa mort [99]. Celle qu'il écrivit pour Philostratos apporte un argument indirect en faveur de liens créés avec Hérode. La probabilité d'une visite de Crinagoras à Jérusalem repose sur les voyages d'Hérode : le roi aurait pu rencontrer le poète soit à Rome, où Crinagoras vivait dans I'entourage d'Octavie, soit dans sa patrie, à Lesbos, lors de la visite qu'Hérode rendit à Agrippa pendant son séjour en Asie Mineure, dans les années 23-21; Crinagoras aurait pu revenir alors à Mytilène dans l'entourage d'Agrippa [100].

Un autre Grec, le brillant orateur Eirénaios, fut peut-être le précepteur d'Antipas fils d'Hérode, futur tétrarque de Galilée, dont il défendit les droits successoraux devant Auguste, de concert avec Ptolémaios de Damas, frère de Nicolas. Eirénaios et Ptolémaios obtinrent pour leur protégé le quart du royaume de son père [101]. Les recherches onomastiques récentes montrent qu'Eirénaios n'est pas un nom sémitique traduit en grec, mais un nom grec authentique, tout normalement dérivé du nom de la paix ; il n'est pas nécessaire de supposer une origine alexandrine à ce personnage d'après la rareté de son nom [102], car celui-ci est bien représenté en Carie et dans le SudOuest de I'Asie Mineure [103].

Enfin, I'hypothèse d'un séjour de Strabon à Jérusalem est envisageable [104], car la probabilité de contacts

[99] BRAUND 1984, ROLLER 2003, p. 87-88.

[100] $A J, 15,350$.

[101] GJ, II, $21 ; A J, 17,226$. SCHALIT 1968, p. 42, «Eirenaios ».

[102] C'est ce que fait Duane W. Roller, qui pense retrouver un Alexandrin dans chaque intellectuel éminent. Il faut revenir à I'hypothèse d'OTTO 1913, col. 90 - suivi par SCHÜRER et al. 1973, p. 311 -, pour qui Eirénaios était un rhéteur grec.

[103] SèVE 2017.

[104] RoLLER 2015, contra, DUECK 2000, p. 190, n. 52, p. 195 , n. 102 , p. 202 , n. 16. 
entre Strabon et Nicolas de Damas transparaît du récit d'une ambassade indienne auprès d'Auguste en 20 av. J.-C., à laquelle les deux historiens ont assisté [105]. Les relations de Strabon avec Hérode ont pu s'établir dans les années 40, alors que Strabon était le protégé de Timagène, lui-même client d'Asinius Pollion ; les contacts auraient pu reprendre quand Strabon accompagna I'expédition d'Égypte conduite par Ælius Gallus en 26-25 [106]. Il faut noter cependant que, si Strabon est passé par Jérusalem en allant en Syrie, il n'a pas fréquenté la cour d'Hérode suffisamment longtemps pour s'intéresser au contexte juif, avec lequel il révèle son manque de familiarité en faisant du roi un grand prêtre [107], ni pour évoquer la reconstruction monumentale du temple de Jérusalem : de tout le somptueux programme architectural dont Hérode avait voulu faire sa vitrine, Strabon ne mentionne que Jéricho et sa palmeraie [108]. En ne retenant de la Judée que le motif emblématique de la Judaea capta, le palmier, I'historien traduit envers Hérode et les Juifs un manque d'intérêt, sinon un dédain, entièrement conforme aux stéréotypes romains [109].

Nonobstant l'ignorance de l'historiographie romaine, la présence massive de lettrés grecs et romains est attestée à la cour de Jérusalem, où la politique culturelle d'Hérode ne se réduisait pas un vernis de culture gréco-romaine judaïsée, mais résultait d'un choix. En témoigne notamment la liste des ambassadeurs nommés par le roi pour exporter l'image de la Judée. Déduite du récit des missions diplomatiques relatées par Flavius Josèphe, cette liste semble dresser le catalogue des lettrés d'origine grecque ou romaine cités plus haut : Andromachos et Gemellus, précepteurs des princes héritiers, Eirénaios I'orateur, autre précepteur probable, Dosithéos le conseiller d'Hérode, Olympos le médecin, Nicolas de Damas le philosophe et son frère Ptolémaios, tous furent chargés de mener des ambassades à Rome. Hérode avait fait le choix de l'ouverture méditerranéenne : sa politique de mixité culturelle s'est traduite jusque dans la création d'un corps diplomatique formé d'intellectuels gréco-romains, qui servirent la Judée et la monarchie hérodienne.

[105] Strabon, Géographie, 15, 1, $73=$ Nicolas de Damas, Histoires, F 100. PAIS 1922, p. 287-288, p. 316.

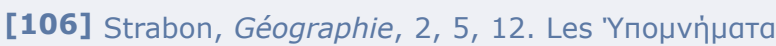
'Iоторıкá furent écrits avant le séjour de Strabon en Égypte avec Ælius Gallus (FGrHist 91, fragments des

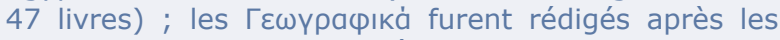
Histoires, comme un supplément et une suite (en 17 livres), mais la date et le(s) lieu(x) de composition de cet ouvrage restent controversés.

[107] Strabon, Géographie, 16, 46 : « Hérode usurpa la charge de grand prêtre et s'y montra tellement supérieur à ses prédécesseurs, surtout dans ses relations avec Rome et dans le gouvernement du pays, qu'il reçut le titre de roi ».

[108] Strabon, Géographie, 16, 41.

[109] Le cliché le plus célèbre en est la plaisanterie d'Auguste déclarant qu'il valait mieux, à Jérusalem, être un porc que le fils d'Hérode, tournant ainsi en dérision à la fois le roi lui-même et les interdits alimentaires des juifs (voir n. 4 ci-dessus).

\section{BIBLIOGRAPHIE}

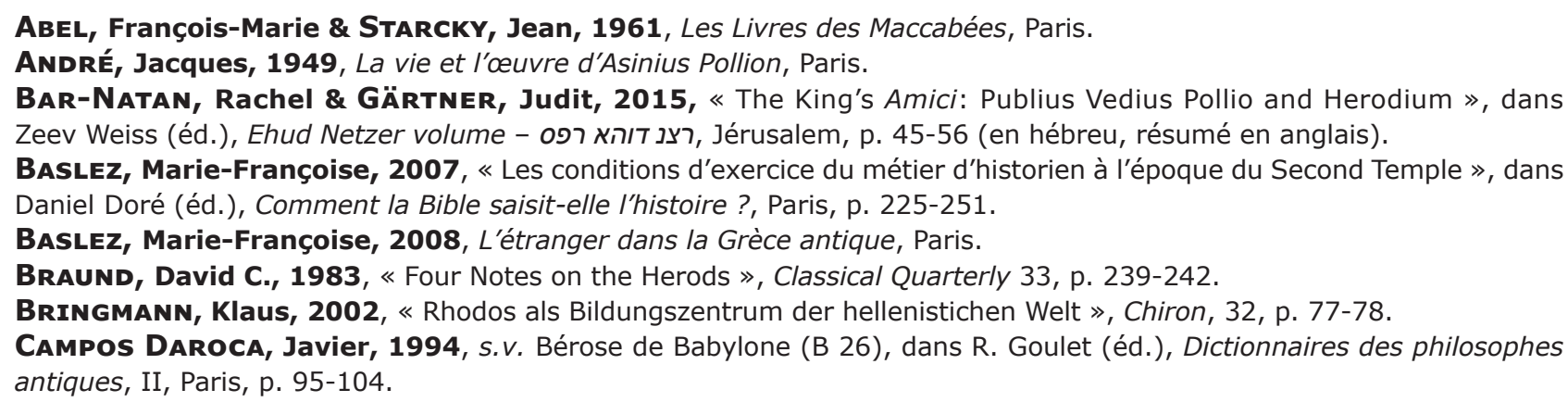

CoARELl, Filippo, 1993, « Bibliotheca Asinii Pollionis », dans Eva M. Steinby (éd.), Lexicon topographicum urbis Romae (LTUR) I, p. 196.

Collins, John J., 1980, «The Epic of Theodotus and the Hellenism of the Hasmoneans », The Harvard Theological Review 73, p. 91-104. 
Coqueugniot, Gaëlle, 2013, Archives et bibliothèques dans le monde grec : édifices et organisation, Ve siècle avant notre ère - II siècle de notre ère, Oxford.

DIHLE, Albrecht, 1959, s.v. Ptolemaios 74, Realencyclopädie der classischen Altertumswissenschaft XXIII, 1, col. 1861. DuECK, Daniela, 2000, Strabo of Amasia: a Greek Man of Letters in Augustan Rome, London.

ECKER, Avner, 2011, « Herod's Workers: The Graffiti Evidence from the Area of the Theater at Herodium - The Greek and Latin Inscriptions », dans David Amit, Guy D. Stiebel \& Orit Peleg-Barkat (éd.), New Studies in the Archaeology of Jerusalem and its Region VI, Jérusalem, p. 194-198 (en hébreu).

ECKer, Avner, 2012, « Homer in Herodium: Graffito of Il. 6.264 », Zeitschrift für Papyrologie und Epigraphik 183, p. $15-20$.

ECKER, Avner, 2015, «A Teacher and Students in Herodion », dans Zeev Weiss (éd.), Ehud Netzer volume - oפר רצי, Jérusalem, p. 6-11.

Feldman, Louis H., 1953, « Asinius Pollio and His Jewish Interests », Transactions of the American Philological Association 84 , p. 73-80.

Feldman, Louiłs H., 1965, Flavius Josephus. General index to volumes I-IX, Cambridge - London.

Feldman, Louis H., 1985, « Asinius Pollio and Herod's Sons », Classical Quarterly 35, p. 240-243.

FGrHist = JАсовY, Felix, Die Fragmente der griechischen Historiker, Leiden, 1926.

FinkielszTejN, Gérald, 2006, «P. Vedius Pollio, producteur de vin à Chios et à Cos et fournisseur d'Hérode le Grand », dans Dariusz Długosz (éd.), Grecs, Juifs, Polonais : à la recherche des racines de la civilisation européenne, Paris (Annales. Centre Scientifique de l'Académie des Sciences Polonaises à Paris, 2, n. spécial), p. 123-139.

GAGgero, Gianfranco, 2011, « Tolomeo, il fratello di Nicolao Damasceno » dans Francesca Gazzano, Gabriella Ottone \& Luigi Santi Amantini (éd.), Ex fragmentis per fragmenta Historiam tradere, Tivoli - Roma, p. 185-196.

GeIger, Joseph, 2002, « Language, culture and identity in Ancient Palestine », dans Erik Nis Ostenfeld (éd.), Greek Romans and Roman Greeks. Studies in Cultural Interaction, Aarhus, p. 233-246.

Gerger, Joseph, 2009, «The Autobiography of King Herod », Judea and Samaria Research Studies 18, p. 79-84 [en hébreu, résumé en anglais].

GeIger, Joseph, 2014, Hellenism in the East: Studies on Greek Intellectuals in Palestine, Stuttgart.

GGM = MüLLER, Karl Otfried, 1851-1928, Geographici Graeci Minores, 9 vol., Paris.

GLAJJ = STERN, Menahem, 1976-1984, Greek and Latin authors on Jews and Judaism, Jérusalem.

GoOdMAN, Martin, 2011, Rome et Jérusalem, Paris.

GREFFCKEN, Johannes, 1922, s.v. Krinagoras, Realencyclopädie der classischen Altertumswissenschaft XXII, col. $1859-1864$. Gruen, Erich S., 1998, Heritage and Hellenism. The Reinvention of Jewish Tradition, Hellenistic Culture and Society, Berkeley.

GünTHER, Linda-Marie, 2005, Herodes der Grosse, Darmstadt.

HARRIS, Harold A., 1976, Greek Athletics and the Jews, Cardiff.

Hengel, Martin, 1996, Judaica et Hellenistica, Tübingen.

HiRschfeld, Yizhar, 2006, « The Library of King Herod in the Northern Palace of Masada », Qadmoniot 113, p. 20-24 (en hébreu).

HolladaY, Carl R., 1989, Fragments from Hellenistic Jewish Authors. Vol. II, Poets: the epic poets Theodotus and Philo and Ezekiel the Tragedian, Atlanta.

ILAN, Tal, 2000, Lexicon of Jewish Names in Late Antiquity. Part I: Palestine 330 BCE-200 CE, Tübingen.

Jacobson, David M., 1988, « King Herod's "Heroic" Public Image », Revue Biblique 85, p. 386-403.

JACOBSON, David M., 2001, «Three Roman Client Kings: Herod of Judaea, Archelaus of Cappadocia and Juba of Mauretania », Palestine Exploration Quarterly 133, p. 22-38.

Kirbihler, François, 2012, s.v. Pollio (P. Vedius) (P 224), dans Richard Goulet (éd.), Dictionnaire des philosophes antiques V b, Paris, p. 1206-1210.

KokкINos, Nikos, 2010, The Herodian Dynasty: Origins, Role in Society and Eclipse, $2^{\mathrm{e}}$ éd. (1 ${ }^{\text {re }}$ éd. 1998), London.

Kuhrt, Amélie \& Sherwin-White, Susan, 1987, Hellenism in the East, London.

LANfranchi, Pierluigi, 2006, L’Exagogé d'Ezéchiel le Tragique, Leiden.

LAQUEUR, Richard, 1936, s.v. Timagenes, Realencyclopädie der classischen Altertumswissenschaft VI A, col. 1063-1071.

LIEBermAN, Saul, 1950, Hellenism in Jewish Palestine. Studies in the Literary Transmission, Beliefs and Manners of Palestine in the I Century B.C.E.-IV Century C.E., New York.

Mussies, Gerard, 1994, « Jewish Personal Names in Some Non-Literary Sources », dans Jan Willem Van Henten \& Pieter Willem Van der Horst (éd.), Studies in Early Jewish Epigraphy, Leiden, p. 242-276.

NA'AMAN, Nadav, 2006, «The Temple Library and the Composition of the Books of Kings », dans André Lemaire (éd.), Congress Volume: Leiden 2004. XVIII th congress of the International Organization for the Study of the Old Testament, Leiden, p. 129-156.

Nesselrath, Heinz-Günther, 1990, Die attische Mittlere Komödie, Berlin.

Netzer, Ehud et al., 2010, «Preliminary report on Herod's mausoleum and theatre with a royal box at Herodium », Journal of Roman Archaeology 23, p. 84-108.

NeTzer, Ehud et al., 2013, « Herodium », dans Silvia Rozenberg \& David Mevorah (éd.), Herod the Great. The King's Final Journey, Jérusalem.

Nicolet, Claude, 1988, L'Inventaire du monde. Géographie et politique aux origines de l'Empire romain, Paris. Oтто, Walter, 1913, s.v. Herodes 14, Realencyclopädie der classischen Altertumswissenschaft supp. II, col. 1-200.

PAIS, Ettore, 1922, «Intorno al tempo ed al luogo in cui Strabone compose la geografia storica», Italia Antica. Ricerche di storia e di geografia storica, vol. 1, Bologna, p. 267-316. 
Parmentier, Édith, 2002, « Les fragments de Denys d'Halicarnasse attribués à Nicolas de Damas. Recherches sur la composition des Excerpta constantiniens », dans Sylvie Pittia (éd.), Fragments d'historiens grecs : autour de Denys d'Halicarnasse, Rome (Collection de l'École française de Rome 298), p. 461-479.

Parmentier, Édith, 2015, « L'énigmatique Anthologie de Stobée », dans François Brizay \& Véronique Sarrazin (éd.), Érudition et culture savante de l'Antiquité à l'époque moderne, Rennes, p. 19-35.

Parmentrer, Édith, 2017, « Le cercle d'Hérode : enquête prosopographique. Identités ethniques et choix politiques dans I'entourage du roi », dans Anne Queyrel Bottineau \& Marie-Rose Guelfucci (éd.), Conseillers et ambassadeurs dans I'Antiquité, Dialogues d'Histoire Ancienne supp. 17, p. 423-452.

Parmentier, Édith \& Barone, Francesca P., 2011, Nicolas de Damas, Histoires, Recueil de coutumes, Vie d'Auguste, Autobiographie, Paris.

Patrich, Joseph, 2009, « Herodian Entertainment Structures », dans David M. Jacobson \& Nikos Kokkinos (éd.), Herod and Augustus: Papers presented at the IJS Conference, Leiden, p. 181-213.

PATRICh, Joseph, 2011, Studies in the Archaeology and History of Caesarea Maritima: caput Judaeae, metropolis Palaestinae, Leiden.

PCG = KASSEL, Rudolf \& Austin, Colin, 1983-1990, Poetae Comici Graeci, Berlin.

Puccr Ben Zeev, Miriam, 1998, Jewish Rights in the Roman World, Tübingen.

RAHMANI, Levy Yizhaq, 1994, A Catalogue of Jewish Ossuaries, Jérusalem.

RICHARDSON, Ernest C., 1963, Biblical Libraries. A Sketch of Library History from 3400 B.C. to A.D. 150, Hamden.

RIESE, Alexander (éd.), 1878, Geographi Latini Minores, Heilbronn (réimpr. 1995).

RoccA, Samuel, 2008, Herod's Judaea. A Mediterranean State in the Classical World, Tübingen.

RoddAz, Jean-Michel, 1984, Marcus Agrippa, Rome (Bibliothèque des Écoles Françaises d'Athènes et de Rome 253).

RolleR, Duane W., 1998, The Building Program of Herod the Great, Berkeley.

RoLler, Duane W., 2003, The World of Juba II and Kleopatra Selene, New York - London.

Roller, Duane W., 2004, Scholarly Kings: The Writings of Juba II of Mauretania, Archelaos of Kappadokia, Herod the Great and the Emperor Claudius, Chicago.

SchalIT, Abraham, 1968, A Complete Concordance to Flavius Josephus edited by K.H. Rengstorf, Supp. 1. Namenwörterbuch zu Flavius Josephus, Leiden.

Schalit, Abraham, 2001, König Herodes, der Mann und sein Werk, Berlin (1 $1^{\text {re }}$ éd. 1962).

SChNeIDeR, Jean-Pierre, 2005, s.v. Nicolas de Damas (N 45), dans R. Goulet (éd.), Dictionnaire des philosophes antiques, IV, Paris, p. 669-679.

SchÜRER, Emil et al., 1973, The history of the Jewish People in the Age of Jesus Christ (175 B.C.- A.D. 135), vol. I, Edimburgh.

SChWARTZ, Daniel R., 2008, 2 Maccabees. Commentaries on Early Jewish Literature, Berlin - New York.

SÈVE, Michel, 2018, «Le nom Irénée, dans la province d'Asie et ailleurs », dans Agnès Bastit (éd.), Irénée de Lyon entre Asie et Occident, Turnhout, p. 91-97 (sous presse).

Sirinelli, Jean, 1993, Les Enfants d'Alexandre. La littérature et la pensée grecques, 334 av. J.-C.-529 ap. J.-C., Paris. SoRDI, Marta, 1982-1983, «Timagene di Alessandria: uno storico ellenocentrico e filobarbaro », Aufstieg und Niedergang der römischen Welt II, 30 , p. 775-797.

Sullivan, Richard D., 1980, « The Dynasty of Cappadocia », Aufstieg und Niedergang der römischen Welt II, 7, 2, p. $1149-1161$.

SullivaN, Richard D., 1990, Near Eastern Royalty and Rome, 100-30 BC, Toronto.

Syme, Ronald, 1961, «Who was Vedius Pollio », Journal of Roman Studies 51, p. 23-30.

VAN DER HORST, Pieter Willem, 2001, «Greek in Jewish Palestine in Light of Jewish Epigraphy », dans John J. Collins \& Gregory E. Sterling (éd.), Hellenism in the Land of Israel, Notre Dame, p. 154-174.

VANDERKAM, James C., 2001, «Greek at Qumran », dans John J. Collins \& Gregory E. Sterling (éd.), Hellenism in the Land of Israel, Notre Dame, p. 175-181.

Vermes, Geza, 2014, The True Herod, London - New York.

WACHOLDER, Ben Zion, 1961, « Greek Authors in Herod's Library », Studies in Bibliography and Booklore 5, p. $102-109$. WACHOLDer, Ben Zion, 1962, Nicolaus of Damascus, Berkeley - Los Angeles.

WeIss, Zeev, 2015, «Buildings for mass entertainment in Herodian Jerusalem: text and artifactual remains, fact or fiction? », dans Zeev Weiss (éd.), Ehud Netzer volume - רצנ דוהא רפס, Jérusalem, p. 104-114 (en hébreu, résumé en anglais). ZecchrnI, Giuseppe, 1982-1983, « Asinio Pollione: Dall'attività politicale alla riflessione storiographica », Aufstieg und Niedergang der römischen Welt II, 30, p. 1265-1296. 